Meta

Journal des traducteurs

Translators' Journal

\title{
Virtualité et textualité en traduction
}

\section{Estella Klett}

Volume 28, numéro 1, mars 1983

La traduction dans le monde

Translation around the World

URI : https://id.erudit.org/iderudit/003325ar

DOI : https://doi.org/10.7202/003325ar

Aller au sommaire du numéro

Éditeur(s)

Les Presses de l'Université de Montréal

ISSN

0026-0452 (imprimé)

1492-1421 (numérique)

Découvrir la revue

Citer cet article

Klett, E. (1983). Virtualité et textualité en traduction. Meta, 28(1), 48-56.

https://doi.org/10.7202/003325ar d'utilisation que vous pouvez consulter en ligne.

https://apropos.erudit.org/fr/usagers/politique-dutilisation/ 


\section{VIRTUALITÉ ET TEXTUALITÉ EN TRADUCTION}

ESTELA KLETT

La thèse que Georges Mounin a soutenue sur «Les problèmes théoriques de la traduction" analyse à fond les difficultés rencontrées dans ce domaine, difficultés que Dominique Aury, dans la préface de ce même ouvrage, symbolise par «les pierres renversées» ou «les peaux de banane» qui entravent le parcours du traducteur.

En effet lorsque l'on décide de traduire dans une autre langue on se sent harcelé par la problématique posée par les deux langues en contact. On ne peut pas se limiter à la connaissance profonde des deux langues que ce soit au niveau morphosyntaxique ou lexical, mais aller beaucoup plus loin et chevaucher bien d'autres domaines. Une initiation aux spécialités neuves (ou vieilles, mais relancées ou rebaptisées) de la linguistique générale comme : la psycholinguistique, l'ethnolinguistique, l'anthropologie linguistique et surtout la socio-linguistique, s'impose.

L'apport des sciences citées ci-dessus, que nous analyserons en détail au long de notre exposé, est tellement riche et divers qu'une analyse hâtive pourrait nous induire à penser que la complication voire une certaine sophistication semble prévaloir sur la simplicité et la clarté. Mais, au nom de la dite clarté, oserait-on négliger les connotations socio-culturelles, les sous-entendus historiques, les colorations propres du terroir, l'affectivité cachée du message, l'évocation suggérée, enfin tout ce qui à l'état virtuel est pétri et malaxé pour fleurir en une signification? Certainement pas, car, si le chemin est plus long, il est aussi beaucoup plus sûr. On aura gagné en fidélité et peut-être parviendra-t-on à éloigner, ne serait-ce que d'un cran, l'anathème qui pèse sur tout traducteur : «traduire c'est trahir».

Nous nous proposons d'analyser successivement les apports des sciences satellites de la linguistique et dont la traduction bénéficie ouvertement.

\section{LE SAVOIR LINGUISTIQUE}

De nos jours il paraît difficile de mener à bien un travail comparatif entre deux langues si l'on méconnait les progrès de la linguistique, qu'elle soit structurale, distributionnelle ou générative.

Si nous remontons à Ferdinand de Saussure, dans son Cours de linguistique générale, nous constaterons que l'illustre genevois dénonçait déjà de son temps la conception simpliste selon laquelle «la langue, ramenée à son principe essentiel, est une nomenclature, c'est-à-dire une liste de termes correspondant à autant de choses». Mais il pousse encore plus loin son idée et ses réflexions 
concernent directement la traduction. Il écrit : «Si les mots étaient chargés de représenter des concepts donnés d'avance, ils auraient chacun, d'une langue à l'autre, des correspondants exacts pour le sens : or il n'en est pas ainsi ». Ce postulat fondamental de Saussure a été repris par beaucoup d'autres linguistes sous des noms très divers : Martinet dans Eléments de linguistique parle de "langue répertoire», Harris dans Distributional Structures parle de «bag of words » ou sac de mots, enfin B.L. Whorf dans Language cite «a piling up of lexations » ou un empilement de mots.

C'est encore Saussure qui explique pourquoi une correspondance exacte entre les termes d'une langue et d'une autre est pratiquement impossible, mis à part les cas de quelques catégories assez spécifiques telles que : les animaux, les arbres, les fruits, les fleurs, les herbes et encore! car souvent à certains éléments de la liste correspond un zéro dans l'autre ou, ce qui est pire, un produit hybride, fruit des croisements, et/ou ayant subi les influences écologiques et climatiques du pays. Sans puiser dans des cultures tout à fait exotiques et très différents, il suffit de prendre un exemple de notre pays pour illustrer ce que nous venons de dire : au marché on trouve en ce moment : "mandarinas», «bergamotas» et «dancis».

Comment établit-on la correspondance avec les «mandarines», les «clémentines » et les «wilkines»?

Le signifié d'un mot recouvre une zone conceptuelle dans une langue, et cette zone n'est pas forcément la même dans l'autre langue. Il peut y avoir superposition totale (c'est rare), chevauchement à des degrés différents (avec petite, moyenne ou grande zone partagée) ou deux zones conceptuelles indépendantes sans aucun point en commun. Les contours du signifié étant, en plus, flous et changeants dans chaque langue, une correspondance exacte, même pour des synonymes, s'avère imparfaite. Ainsi, les paires «cheval» et «coursier» ou «porc» et ««cochon» (le même exemple est valable pour l'espagnol: «caballo» et «corcel»; «cerdo», «puerco» et «chancho») qui recouvrent apparemment la même surface conceptuelle n'ont jamais pu fonctionner dans les mêmes contextes de manière interchangeable: «donnez-moi une tranche de porc» «il a fait un travail de cochon». La substitution de «porc»par «cochon» et vice versa engendre des phrases incorrectes: * «une tranche de cochon", * "un travail de porc», ne sont pas acceptables. Un peu plus loin dans cet exposé, on trouvera une étude du sens sous la rubrique "Sémantique et traduction». C'est dans l'analyse y proposée que nous élargirons les recherches sur les contours du signifié. C'est dans ce contour souple et estompé que la connotation littéraire de «coursier» (ou «corcel» en espagnol) trouve sa place. Les sphères de signification des deux mots ne peuvent plus s'enchâsser dans le même puzzle.

D'autres faits langagiers nous démontrent à quel point une réflexion linguistique profonde est indispensable quand nous devons rendre un texte dans une autre langue.

Lorsque je traduis la phrase: «Se iba haciendo tarde» par «Il se faisait tard peu à peu», $\mathrm{j}$ 'accomplis une démarche linguistique car alors que l'idée de progression dans le temps l'espagnol l'exprime grâce à la forme composée du 
gérondif : «iba haciendo», le français fait retomber ce mouvement sur l'adverbe. Il en est de même pour faire passer l'idée de progression dans l'espace : «andaba buscando libros»= «je cherchais des bouquins ça et là».

Ce sera la classe des adverbes ou un adjectif ou le lexème lui-même, qui nous aidera en français à suppléer à la permanence ou à la momentanéité évoquée par les verbes «ser» ou «estar» de l'espagnol. «Es nerviosa» $\neq$ «está» nerviosa»: «elle est nerveuse» $\neq$ «elle est énervée»; «eres rico» $\neq$ «estás rico»: «tu es riche» $\neq$ «te voilà riche»; ou dans une conversation plus familière: «estoy viuda» s'opposant à «soy viuda». Il s'agit une fois de plus de jouer sur la forme adverbiale pour traduire en français la virtualité du veuvage, «je suis célibataire pour quelques jours» = «momentanément», "occasionnellement ». En anglais c'est le substantif en lui-même qui est porteur de cette notion de fugacité qui s'oppose à la pérennité de l'état, "a widow» s'oppose donc à «a grass-widow» «ser viuda» et «estar viuda»).

Le français, assure-t-on, est une langue qui excelle à nominaliser alors que l'espagnol s'affirme en accordant au verbe une grande puissance. "Au travail!» («au boulot!» en français populaire) ou «aux armes!» (comme dans les célèbres strophes de la Marseillaise) donnent en espagnol: «a trabajar!» («a laburar!» en lunfardo) «a luchar!», «a pelear!». Cependant la forme nominale, bien que moins fréquente, coexiste en espagnol avec la forme verbale dans d'autres locutions qui ont également une valeur d'intimation. C'est le cas de : «au lit!» que l'on traduit par: «a la cama!», ou: «à dormir!» et «à table!» qui donne: «a la mesa!» et «a comer!».

Un exemple semblable est celui qui nous est proposé par la signalisation routière: la pancarte française porte l'écriteau suivant: «travaux», celle d'Argentine: «gente trabajando».

On pourrait d'ailleurs s'amuser à faire un relevé des manchettes d'un journal français et argentin pour constater que les nominalisations font pendant aux verbes conjugés, noyaux de phrase, ou constituants de phrase à eux seuls. «Subió el dolar», «Galíndez mató», «detienen a un narco-traficante», «llama el gobierno al canciller argentino en Santiago ». Les nouvelles qui inquiètent ou rassurent l'Argentine n'étant pas les mêmes de France à une date précise, nous n'avons pas pu vérifier comment les gros titres cités auparavant, étaient traduits en français. Mais nous en avons relevé d'autres aussi significatifs : "Augmentation du prix du pétrole»; «l'agonie du monopole»; «coup de fouet du patronat»; «la santé publique en danger»; «Kaboul le fric»; «Désinformation permanente».

Il faut se méfier des généralisations hâtives mais il est bien certain que dans tous les exemples montrés le verbe l'emporte en espagnol.

La désinence verbale se trouve à droite en espagnol et à gauche en français (comme pour beaucoup d'autres faits linguistiques, d'ailleurs).

En effet les formes «canto, cantas, cantamos...» etc. de l'espagnol contrastent avec la forme orale du français [ft] qui correspond à: «je», «tu», «il», «elle", "on» et «ils, elles». D'où la nécessité en français d'ajouter des pronoms sujets pour dissiper l'ambiguïté du code oral. Par contre, l'utilisation des mêmes pronoms en espagnol relèverait de l'emphase. «Il le fera de bon gré» donne 
«Lo hará de buen grado» et non pas «El lo hará de buen grado» qui serait une forme marquée par rapport à l'énoncé de départ.

Il en est de même pour l'utilisation, très fréquente, en français aussi bien qu'en anglais, des possessifs. Dans les exemples qui suivent aucun possessif n'est traduit en espagnol par un possessif.

— «Je n'ai pas pu joindre Jean au téléphone, mais j’ai eu sa sœur.» Ou au téléphone : «Allô? c'est Marion? - Non, c'est sa mère.»

- «J'ai mis mon chapeau sur ma tête.» Prévert in Déjeuner du matin.

- Au marché : «Regardez mes belles pommes!"

- L'apostrophe pour s'adresser aux religieux : «mon père», «ma mère», «ma sour", «mon frère ».

- «mon train, je l'ai raté ce matin et comme il était déjà trop tard je n'ai pas pu acheter mon journal.

- «Qu'est-ce qu'elle fait ta fille Hélène? - Elle fait sa médecine (son droit).

Nous ne nous proposons nullement de faire l'inventaire exhaustif des faits linguistiques qui intéressent l'espagnol et le français car il y a risque d'interférence; nous voulons plutôt démontrer qu'on peut traduire en dépit des différences, parfois frappantes, des syntaxes.

C'est peut-être cette constatation qui a poussé certains linguistes à parler des universaux de syntaxe, universaux qui se trouveraient en structure profonde, malgré l'hétérogénéité de surface (cf. Chomsky, Syntactic Structures). Malheureusement les recherches dans ce domaine en sont à l'état embryonnaire et on a seulement tracé des schémas très généraux des grandes fonctions et relations communes à deux ou plusieurs langues en contact.

Il faudra une certaine sensibilité linguistique, ne serait-ce qu'intuitive, pour accepter que le pluriel dans "Nous sommes allés sur les montagnes russes» naît d'une «vision du monde différente», pour reprendre une expression de Whorf. Ainsi l'espagnol plutôt que de voir la suite de montées et de descentes du jeu des parcs d'attractions, concentre son attention sur l'ensemble de l'engin, ce qui donne, en toutes circonstances, «la montaña rusa».

Le thème de la «vision» du monde, ou du «découpage du réel» qui change selon les cultures, même les pays, a été suffisamment développé pour qu'on lui consacre une analyse supplémentaire. Nous voulons simplement montrer le rapport étroit entre le fait linguistique et l'univers de notre expérience, tel que Wilhelm von Humboldt et ses descendants l'ont envisagé. "Tout système linguistique renferme une analyse du monde extérieur qui lui est propre et qui diffère de celle d'autres langues». Ainsi si nous avions à traduire: "Attention, le feu est orange!», bien que l'espagnol possède «naranja», comme notre vision du feu est différente, il faudra dire «amarillo» et si nous nous trouvions au Pérou «ambar». De même pour le mot «cáscara» de l'espagnol. La consistance de celle-ci ne joue aucun rôle chez nous alors que les français «voient» et séparent les «cáscaras» en fonction de leur épaisseur et leur degré de dureté. Ce faire est naturellement automatique et intuitif pour tous les locuteurs francophones. On a ainsi «des épluchures», «des peaux», «des pelures», «des croûtes», «des coquilles», «des coques», «des écales», «des écorces»; tous ces mots englobés par «cáscara» en espagnol.

C'est encore du domaine linguistique que de sentir que le gérondif espagnol «circulando!», «andando!» ou «corriéndose al interior!» a une valeur impéra- 
tive et devra être traduit en français par l'impératif : circulez! allez! poussezvous!

Sans ériger l'échantillonnage d'interférences décrites précédemment en vérité générale, il est indéniable que le choix des moyens formels d'expression passe par un tamis linguistique.

Mais ce serait tomber dans une autre erreur aussi grave que l'ignorance des règles linguistiques que de vouloir enfermer la traduction, ses problèmes et ses solutions dans les frontières de la linguistique au moins au sens étroit du terme.

La traduction étant une série d'opérations de l'esprit dont le point de départ et le produit final sont des significations, elle pourra avoir recours à la linguistique dans les opérations intermédiaires plus formelles.

Mais on ne saurait en rester là, on devra dépasser le stade de la structuration linguistique rigide pour tremper l'énoncé dans la souplesse sémantique où des lois de structuration inattaquables scientifiquement n'ont pas été découvertes. C'est seulement à ce moment-là qu'on aura percé l'obscurité et qu'une signification jaillira.

\section{AU-DELÀ DU SENS : EN QUÊTE D'UNE SIGNIFICATION}

\section{Sémantique et traduction}

Bien que Saussure n'ait pas utilisé le mot sème, il y a dans ses «Cahiers» des notes concernant son emploi.

C'est Peirce qui, lors d'une discussion sur les racines de l'algèbre, la logique et la sémiologie, exprima le besoin de substituer quelques mots très habituels par d'autres chargés d'un nouveau contenu.

Mais c'est sans doute chez Buyssens et puis chez Prieto que ce concept se développe considérablement. Enfin Greimas et Pottier analysent jusque dans leurs moindres détails les composantes du sens.

Greimas, s'appuyant sur les idées de Hjemslev d'une part et celle de Jakobson d'autre part, donne le nom de sème aux «traits distinctifs» du signifié selon Jakobson (ceux-là sont à leur tour, les «éléments différentiels » de Saussure).

Dans un énoncé quelconque il y a, dans un signifié pris comme exemple, des constantes invariables du signifié. C'est un «minimum sémique que Greimas appelle «noyau sémique» (NS).

Au cours de l'actualisation on mobilise diverses variables sémiques qui donnent «l'effet du sens», Greimas les appelle «des sèmes contextuelles » (NS) (unité minimale de signification elle aussi). L'addition du NS (noyau sémique) et du CS (sèmes contextuels) donne l'effet de sens qui est appelé sémème.

Selon Pottier quand on réfléchit sur l'expression significative d'un morphème lexical quelconque on voit qu'il appartient à un ensemble infini: c'est l'ensemble de zones sémantiques.

Mais quand ce morphème lexical s'actualise dans l'acte de la communication, il fonctionne dans un domaine déterminé. Sa signification est, donc, relative, parce qu'elle prend telle ou telle autre extension en fonction des circonstances de l'acte de communication. La situation est donc décisive et le mor- 
phème lexical n'aura pas de signification tant qu'il ne se sera pas imprégné dans le contexte circonstanciel. Ayant ainsi grignoté une nouvelle extension ou s'étant emparé de colorations supplémentaires, le morphème lexical devient un sémème (cf. Pottier).

Ce sémème peut être décomposé dans ses parties constituantes parce que quelques unes sont décisives pour la réalisation de la signification dans toutes les circonstances tandis que d'autres ne le sont pas toujours. On peut classer ces composantes, auxquelles Pottier donne le nom de «sèmes», comme il suit:

- Des sèmes spécifiques qui font partie du mot isolé ou des expressions de manière telle que l'on peut distinguer un mot d'un autre, ou une expression d'une autre, quoiqu'ils soient très voisins dans la zone sémantique. Ils constituent le «nucléus» du sème ainsi conçu. Ils représentent les définitions de la signification dans les bons dictionnaires. Naturellement ils peuvent faire partie de certains traits très concrets dans les objets ou les actions, ou bien, ils peuvent être le résultat d'un processus cognitif dans certains mots abstraits.

C'est un sème spécifique qui nous permet de distinguer «mourir» de «décéder», qui implique obligatoirement «humain» et non pas animal. Le même exemple est valable en espagnol «morir» et «fallecer». La distinction entre «vivant» et «mort» est bien connue en anglais ou en espagnol pour les animaux : «cow» «beef», «sheep» «mutton», «pig» «pork», et «pez» «pescado». Les humains y sont sensibles en français grâce à la combinatoire de la phrase :

«j'ai beaucoup d'amitié pour ton père (il est vivant).

«j'ai beaucoup d'admiration pour ton père (il est vivant ou mort).

- Des sèmes génériques qui permettent l'extension de la signification puisqu'ils comprennent certains traits à l'intérieur de la même classe conceptuelle. Ils constituent la base commune des parasynonymes et ils peuvent se trouver, si l'on fait une représentation graphique, dans la périphérie du «nucléus» constitué par les sèmes spécifiques.

-- Des sèmes virtuels. Ils sont le résultat d'un usage occasionnel du morphème lexical. Si les sèmes génériques constituent la périphérie, les sèmes virtuels sont des bourgeons virtuels de cette périphérie, comme ils peuvent être aussi des embryons potentiels des sèmes spécifiques puisqu'ils naissent aussi de l'utilisation figurée, métaphorique des sèmes spécifiques.

Si nous nous sommes attardés quelque peu sur la notion de sème c'est qu'il résulte presque nécessaire de ce qui précède que le traducteur ne pourra en aucun cas ni nier, ni écarter, ni esquiver les difficultés qui apparaissent quand dans l'acte de communication tel ou tel virtuème (sème virtuel) s'est réalisé ayant pris une consistance qui, indubitablement, sert à nuancer ou à affiner le message.

En d'autres termes nous pouvons dire que la connotation est née. Dans la mesure où la dénotation s'appuie sur les sèmes spécifiques et génériques, la connotation dépend de sèmes virtuels.

À notre avis, le plus dur travail du traducteur est celui de saisir pleinement en langue source, dans un premier temps, «les liens sémantiques» (dénomination de Mel'čuk (dans Esquisse d'un modèle linguistique du type sens-texte), c'est-à-dire les relations dynamiques que le mot entretient dans l'énoncé. En ou- 
tre il est nécessaire de sentir profondément quelle est la connotation véhiculée car il y a eu actualisation d'un ou plusieurs atomes ou embryons de sens qui se trouvaient à l'état virtuel. À ce stade on aura dépassé le sens et on aura atteint une signification.

Dans un deuxième temps il s'agit de faire le même travail en langue cible, ce qui est bien plus délicat encore.

Ce double travail de va-et-vient comme si l'on parcourait une voie et on rebroussait chemin lentement, entraine un peu moins de défaillances dans la traduction.

Pour y parvenir il nous faudra donc recréer la situation de départ sachant à l'avance que le mot, l'expression à choisir devront non seulement être fidèles à l'énoncé de base, mais aussi porteurs de scintillement ajouté, dans la communication verbale, par les gestes, les pauses, l'intonation. C'est là un premier avatar.

Puis on s'attaquera à explorer le champ sémantique, à tâtonner, et, par des approximations successives, à sentir que certains nœuds du réseau sémantique s'ouvrent ou se débloquent pour laisser surgir l'équivalent le plus juste. Cette opération qui demande du doigté et une extrême souplesse a été très bien décrite par B.F. Ries et par G. Rasran dans Experimental Semantics.

Si nous avons utilisé la terminologie de «réseau» et «nœud» dont on se sert pour les ordinateurs, c'est bien pour montrer que la correspondance rigide des circuits de la machine peut difficilement prévoir à l'avance les appendices du sens (virtuèmes) qui vont être actualisés ou non. Par contre dans le domaine de la science, là où la connotation joue un rôle nul ou presque nul, la traduction automatique est valable et utile. Melnikof a très bien décrit la flexibilité et le dynamisme de l'esprit humain qui contraste avec la rigidité et l'intransigeance de la machine.

Pour avancer avec aisance et souplesse, le traducteur doit connaitre le champ sémantique dans lequel se situe son texte à traduire. Cela implique plusieurs niveaux de connaissance. Au sens large du terme il s'agit de caser la traduction dans le domaine auquel elle appartient.

La signification d'une lexie étant tributaire de l'ensemble dans lequel elle fonctionne, il convient de la situer dans le microchamp d'expérience: le dialogue enfantin, la culture du maïs, les voyages, le rugby, etc. Quelquefois le domaine est 'très spécifique: l'informatique, la géologie, l'astrologie.

Le travail à l'intérieur du microchamp allège le concept de polysémie. Il peut exister des liens interchamps: il faudra les dénicher car ce sont des virtualités qui cachent souvent des jeux de mots ou des expressions ironiques.

Enfin au sens étroit, connaître le champ sémantique signifie répertorier l'ensemble de parasynonymes et de soi-disant synonymes qui tournent autour de l'expression que l'on analyse.

Nous croyons avoir suffisamment développé la notion de virtuème et de connotation pour démontrer qu'il ne peut pas être question de négliger «ces valeurs supplémentaires» comme dit Bloomfield dans Language. Il en fait d'ail- 
leurs un inventaire assez complexe: connotation vulgaire, familière, académique, provinciale, rustique, archaïque, technique, savante, étrangère, ironique, argotique, enfantine.

Il existe plusieurs dénominations pour ces colorations du signe: Charles Morris y voit «des propriétés additionnelles du signe», Ogden et Richards opposent «les significations référentielles» aux «émotives», Buyssens dans Structuralisme et arbitraire confronte «le désignant» et «la valeur», d'autres linguistes classent les signes en "cognitifs et dynamiques» ou "référentiels et évocatifs».

Bien que ces colorations du signe reçoivent des noms divers on y retrouve un dénominateur commun: l'affectivité ou l'expression, le facteur imaginatif du message.

C'est bien charger le mot d'une valeur affective que de dire en espagnol: «Señora, los chicos están bañaditos y cenaditos» ou «Me las va a pagar Varguitas, yo también diré mi verdad» comme s'écria la tante et ex-épouse de Mario Vargas Llosa, doña Julia Urguidé Illanes ou encore les adverbes «rapidito", «todito", "ahorita», "ahoritita», «ahicito», si chers à nos compatriotes des provinces du nord de l'Argentine. L'utilisation fréquente des diminutifs en espagnol cache souvent une tonalité affective plus qu'elle ne veut signifier la petite taille de la chose dont on parle.

C'est surtout lorsqu'il a une valeur motivée que le mot gagne de l'éclat. La motivation est le sentiment qu'a le locuteur que les signes renferment des éléments identifiables. (C'est le cas des onomatopées en général et de beaucoup de mots composés). «Mesa» ou «table» sont arbitraires. «Mesa de luz» ou «table de nuit» sont motivés.

Quand un français dit "un cache-misère» ou un «anti-sèche», le mot prend une saveur qu'il est difficile de lui accorder en traduction. Ainsi «remiendo " pour cache-misère est trop neutre, alors que «rodillera» ou «codera" ou «parche» que l'on dit au moins aux bords du Rio de la Plata, est bien plus près. «Toubib» en français perd par rapport à «matasanos», et se situe vraisemblablement au même niveau de signification que «tordo" en Argentine. Par contre pour «perdre les pédales» et "perder los estribos» la correspondance est optimale.

Viser la connotation véhiculée par l'énoncé est toujours une cible difficile, mais quand l'expression en question s'est imprégnée d'un contenu de civilisation l'obstacle est encore plus grand.

Ainsi quand nous disons en Argentine «enseguida mostró la hilacha», la signification est toute autre que de dire «montrer ce que l'on est». L'expression n'est jamais positive ou neutre mais toujours péjorative. C'est le rappel de Vizcacha dans le Martín Fierro qui revient pour évoquer en filigrane les défauts de «l'antigaucho» ou de «l'anti-héros»: «Me llevó consigo un viejo que pronto la hilacha dejaba ver por su facha que era muy renegado y ladrón».

Pour prendre un exemple français il suffirait de dire: «Ah! Heureux qui comme Ulysse» pour qu'un français d'un niveau culturel moyen puisse reconnaître immédiatement, il me semble, les vers de Joachim du Bellay teints de regrets et de nostalgie. 
Enfin les exemples sont nombreux qui nous prouvent à quel point il faut pénétrer le texte, s'imprégner de lui et être aux aguets des valeurs évocatrices qui lui donnent une signification.

Souvent, d'ailleurs, ce sont ces significations latentes, difficiles à déceler et à fortiori à traduire, qui confèrent au mot, au texte, une certitude magie, un caractère poétique, humoristique ou ludique.

On ne saurait conclure sans revoir deux aspects déjà cités, qui nous paraissent particulièrement intéressants.

Tout d'abord nous insistons sur la nécessité pour le traducteur de «jouer le rôle de l'ethnographe» comme le dit Georges Mounin. Cela revient à posséder la culture totale, si possible, d'une communauté donnée, c'est-à-dire «l'ensemble des activités et des situations par où cette communauté se manifeste (structure et vie sociale, organisation du système des connaissances, droit, religion, morale, activités esthétiques »).

C'est ce que B. Pottier (dans «L'Ethnolinguistique», Langages $\mathrm{n}^{\circ} 18$, juin 1970) appelle «l'étude du message linguistique en liaison avec l'ensemble des circonstances de la communication».

Le lien entre langue et culture est évident dans la mesure où la langue est un code économique de transmission d'une information dans certaines circonstances. Alors les réalités quotidiennes auront de fortes chances d'être exprimées économiquement. C'est pourquoi c'est surtout au niveau des taxinomies exprimées élémentairement que les langues vont se différencier.

Il est rare qu'on ne puisse exprimer dans une langue avec des moyens complexes ce qu'une autre exprime synthétiquement.

Notre but, en traduction, ce sera de trouver le moyen en même temps économique et fidèle au référent.

Cela nous amènera parfois à produire une périphrase libre, non intégrée dans la langue cible. C'est le cas de «faldero» (dans l'énoncé «perro faldero») "qui aime être sur les genoux», ou la traduction proposée à l'affiche pour le film «La dentellière»: «La bordadora de puntillas» ou «encajes».

Ensuite l'autre aspect que nous nous permettrons de privilégier est celui du miroitement du mot (permettez-moi la métaphore!) À la limite tout est connoté (cf. les noms, les chiffres: le 13 porte malheur dans beaucoup de pays civilisés, le 24 est néfaste et péjoratif au Brésil car au «jogo do bicho» le chiffre est attribué au «veado» = cerf (qui désigne l'homosexuel).

Imprégnés par le dynamisme du texte, nous engagerons nos recherches sachant que rien n'est définitif, que nous aurons à polir, à raboter, à façonner sans nous lasser. Tâche colossale que de rendre la langue, cet être vivant qui palpite, qui bouge, qui virevolte, qui escamote, qui estropie.

Si notre texte n'est pas terne et statique, si les mots ne se retrouvent pas bancals, boitilleux et clopinards mais qu'ils frémissent et rayonnent de signification, c'est que nous avons dépassé la nébuleuse de virtualité et que nous sommes près, très près ou dans la textualité. 